\title{
Study on the Viscoelastic Properties of the Epoxy Surface by Means of Nanodynamic Mechanical Analysis
}

\author{
YANG-FEI ZHANG, ${ }^{1}$ SHU-LIN BAI, ${ }^{1}$ DA-YONG YANG, ${ }^{2}$ ZHONG ZHANG, $^{2}$ SHARON KAO-WALTER ${ }^{3}$ \\ ${ }^{1}$ Centre for Advanced Composite Materials, Department of Advanced Materials and Nanotechnology, LTCS, \\ College of Engineering, Peking University, Beijing 100871, China \\ ${ }^{2}$ National Center for Nanoscience and Technology, Beijing 100080, China \\ ${ }^{3}$ Department of Mechanical Engineering, School of Engineering, Blekinge Institute of Technology, \\ SE-371 79, Karlskrona, Sweden
}

Received 29 April 2007; revised 22 October 2007; accepted 26 October 2007

DOI: 10.1002 / polb.21365

Published online in Wiley InterScience (www.interscience.wiley.com).

\begin{abstract}
The viscoelastic properties of the epoxy surface have been investigated by nanodynamic mechanical analysis (nano-DMA). Both a Berkovich tip and a conospherical tip were used under the condition of different forces (i.e., different penetration depths) in the frequency range of $10-200 \mathrm{~Hz}$. Loss tangent and storage modulus are characteristics that describe the viscoelastic properties. The effect of force frequency, penetration depth, and tip shape on the viscoelastic properties is studied and discussed according to the features of microstructures and mobility of molecular chains. The experimental results show important variations when the penetration depth is shallow $(<30 \mathrm{~nm})$. As the depth becomes deeper, the results tend to be stable and become almost constant over $120 \mathrm{~nm}$. The two kinds of indenter tip can cause a slight difference of the storage modulus. A "master curve" of the storage modulus as a function of force frequency is established. ( 2007 Wiley Periodicals, Inc. J Polym Sci Part B: Polym Phys 46: 281-288, 2008
\end{abstract}

Keywords: nano-DMA; resins; surfaces; viscoelastic properties

\section{INTRODUCTION}

Epoxy is one of the most important polymers used for diverse applications such as anticorrosive coatings, encapsulation of microelectronic devices, high-voltage switches, storage batteries, adhesives, and polymer composites. The synthesis of epoxy was discovered as early as the late 1890 s. After that, great progress has been made in the processing and manufacturing of epoxy resins and in the characterization of their properties. ${ }^{1}$ Most cured epoxy resins provide amorphous

Correspondence to: S. L. Bai (E-mail: slbai@pku.edu.cn) Journal of Polymer Science: Part B: Polymer Physics, Vol. 46, 281-288 (2008) (2007 Wiley Periodicals, Inc. thermosets with excellent mechanical properties, outstanding chemical performances, pre-eminent electrical behavior, good corrosion resistance, strong adhesion, and low shrinkage upon cure. These superior performances have gained epoxy resins the extensive adoption in surface engineering and tribological applications, where the surface properties are meaningful for material selection and design improvements. ${ }^{2}$ Since most initial interactions occur at the surface, viscoelastic properties are values that represent the contact performances of material surfaces. ${ }^{3-5}$ The hysteretic effect and time-dependent deformation are usually reflected by the values of the storage modulus, loss modulus, or loss tangent.

Nanodynamic mechanical analysis (nanoDMA) has been used to evaluate the viscoelastic 
properties of various classes of polymers. This method has proven to be an effective method for nanoscale studies. ${ }^{6-13}$ It is based on the dynamic model of depth-sensing indentation which utilizes the load-penetration depth curves to characterize hardness, toughness, Young's modulus, residual stress, yielding stress, and subsurface damage for both film and bulk materials. ${ }^{14-16}$ The widely accepted scheme (Oliver and Pharr ${ }^{15}$ ) was developed from Sneddon's ${ }^{17}$ contact mechanics solution on a linearly elastic halfspace, and it works well for the elastic contact of tip and sample during the unloading period. In the past decade, the DMA method has been used extensively to characterize hard materials such as metals and ceramic films. Recently, soft materials such as polymers were also investigated and the results are in good agreement with those obtained by traditional micro- and macroscale experiments. Several improvements have been made to remove the effect of the time-dependent behavior on elastic modulus measurements of viscoelastic materials. ${ }^{18,19}$ However, the empirical or principal solutions to direct measurements of the time-dependent indentation behavior are more important and desired. ${ }^{20-24}$ With the development of high-resolution testing instruments, the properties measurement of material surfaces at nanometer scales becomes possible and accurate. But additional difficulties are caused by the size effect: the viscoelastic response depends strongly on the imposed contact conditions, defined by competing length scales including material surface roughness, indentation penetration depth, and tip shape. In polymers, parameters like force frequency, loading rate, temperature, and humidity can also strongly affect the viscoelastic response. These interference factors are intentionally eliminated by a control of the experimental conditions. In practical applications, the imposed loading is often dynamic or cyclic, thus it is very difficult to obtain meaningful viscoelastic properties by only using quasi-static indentation or creep indentation. Nano-DMA is newly developed to remedy the insufficiencies of quasi-static testing to display significant timedependant deformation and recovery. ${ }^{25}$ Several experimental studies have been made on polymer surfaces with the use of nano-DMA. For example, Chakravartula and Komvopoulos ${ }^{6}$ investigated the viscoelastic properties of linear polymer surfaces, such as low-density polyethylene, high-density polyethylene, and ultrahigh molecular weight polyethylene. Moreover, White et al. $^{8}$ studied a cured epoxy, a poly(methyl methacrylate), and two poly(dimethyl siloxane) samples of different crosslink densities. The indentation stress fields were studied theoretically as well as by means of photoelastic tests, ${ }^{26}$ and molecular dynamics simulations were newly used to predict the stress fields in the nanometer regime. ${ }^{27}$

The objective of this work is to investigate the near-to-surface viscoelastic properties of a crosslinked polymer: a cycloaliphatic epoxy. Nano-DMA tests were performed on this epoxy by using two types of indenter tips, and by applying different force frequencies and penetration depths. The continuous stiffness measurement $(\mathrm{CSM})^{28}$ tests were undertaken to investigate the variation of viscoelastic properties with the penetration depth.

\section{EXPERIMENTAL}

\section{Materials}

The resin is a commercially available cycloaliphatic epoxy (UVA-CURE1500), hardened by an anhydride curing agent (Albidur HE 600), with the specific equivalent weights of 129 and $170 \mathrm{~g} /$ equiv., respectively. The sample was supplied by hanse chemie AG (Germany) and then made with a thickness of $10 \mathrm{~mm}$ to eliminate the effect of substrate. The surface roughness measured by an atomic force microscope (SPI3800NSPA400 (Seiko)) in contact mode on a scan area of $2.5 \mu \mathrm{m} \times 2.5 \mu \mathrm{m}$ is about $1 \mathrm{~nm}$ Ra. The epoxy has been used as a nanocomposite matrix, and its mechanical properties have been measured by dynamic mechanical thermal analysis, tensile, flexural (three-point bending), and microhardness tests. ${ }^{29}$

\section{Experimental Procedure}

The nano-DMA tests were performed with the use of a nano-DMA II ${ }^{\mathrm{TM}}$ adjunct of TriboIndenter (Hysitron Incorporated) with both a Berkovich diamond tip of a nominal radius of curvature equal to $\sim 100 \mathrm{~nm}$ and a $60^{\circ}$ conospherical diamond tip of $\sim 1 \mu \mathrm{m}$. Two types of dynamic loading processes were undertaken as shown in Figures 1 and 2, respectively. The purpose of the first loading process (Fig. 1) was to obtain the dependence of the viscoelastic behavior on 


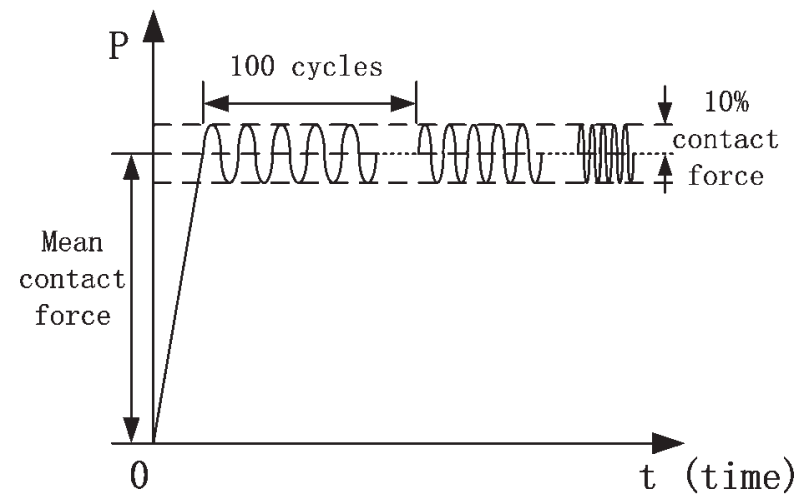

Figure 1. Schematic presentation of the dynamic loading process.

the contact force (or penetration depth). Ten levels of mean contact force were chosen with the values of $7,9,12,15,22,36,80,165,225$, and $250 \mu \mathrm{N}$, respectively. The force increases fast to a mean load, and a frequency sweeps at that load from 10 to $200 \mathrm{~Hz}$ with 100 cycles under each frequency. The tip was then moved to another position with a repetition of the same loading process, and so on. The alternating force was set at a constant value that is equal to $10 \%$ of the mean contact force, to make sure that the amplitude was large enough to obtain reliable data at a high frequency. The second loading process is CSM (Fig. 2). In this case, the load was applied to a value at which the frequency sweep was adopted at fixed $75 \mathrm{~Hz}$ for 100 cycles. The load was then increased to a higher value at the same location of the sample

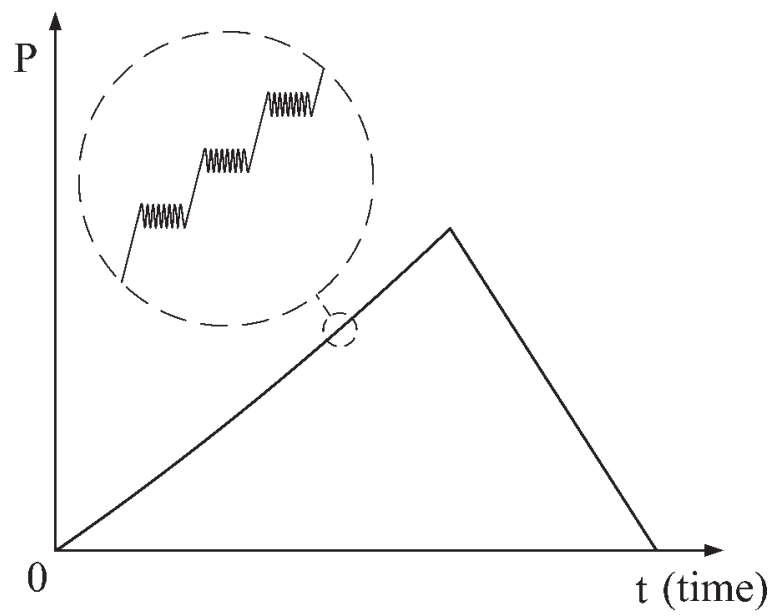

Figure 2. Schematic presentation of the CSM loading process.

Journal of Polymer Science: Part B: Polymer Physics DOI $10.1002 /$ polb with $8.5 \mu \mathrm{N}$ increment, the same frequency sweep was followed, and so on. The load range was from about 10 to $350 \mu \mathrm{N}$. The choice of 75 $\mathrm{Hz}$ was made to avoid the resonant frequency caused by the electric power frequencies in different countries. For both loading processes, 20 indents were made under each load to obtain average values of the storage modulus and the loss tangent. During the experiments, the temperature was controlled at $\sim 27{ }^{\circ} \mathrm{C}$ and the relative humidity was $43 \pm 3 \%$.

\section{Dynamic Mechanical Analysis}

During the experiments, a capacitive load-displacement high-resolution DMA transducer was used. The load resolution is $1 \mathrm{nN}$ and the displacement resolution $0.04 \mathrm{~nm}$. The dynamic model adopted was developed by Asif and coworkers $^{10}$ as shown in Figure 3. Here, $m$ is the mass of indenter, $C_{\mathrm{s}}$ and $C_{\mathrm{i}}$ are the damping coefficients of the sample and the capacitive displacement sensor, $K_{\mathrm{s}}$ and $K_{\mathrm{i}}$ are the stiffness of the sample and the spring holding the indenter, respectively. $C_{\mathrm{i}}$ and $K_{\mathrm{i}}$ are provided by the constant calibration of the equipment at air before testing and embedded in the software analysis. The storage modulus $E^{\prime}$, the loss modulus $E^{\prime \prime}$, and the loss tangent $\tan \delta$ will be obtained by:

$$
\begin{aligned}
E^{\prime} & =\frac{K_{\mathrm{s}} \sqrt{\pi}}{2 \sqrt{A_{\mathrm{c}}}}, \quad E^{\prime \prime}=\frac{\omega C_{\mathrm{s}} \sqrt{\pi}}{2 \sqrt{A_{\mathrm{c}}}}, \\
\tan \delta & =\frac{E^{\prime \prime}}{E^{\prime}}=\frac{\omega C_{\mathrm{s}}}{K_{\mathrm{s}}},
\end{aligned}
$$

where $A_{\mathrm{c}}$ is the projected contact area. Because of the small penetration depths in this work,

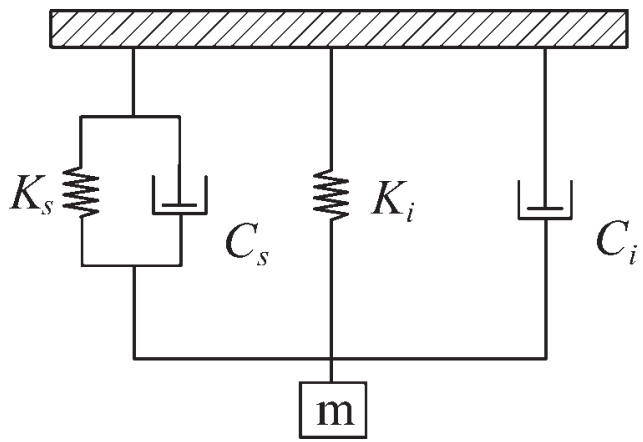

Figure 3. Dynamic model of the tip contacting with the sample. 
fused quartz is used as the standard reference material to calculate $A_{\mathrm{c}}$.

The reduced storage modulus $E_{\mathrm{r}}^{\prime}$ and the reduced loss modulus $E_{\mathrm{r}}^{\prime \prime}$ can be read directly from the equipment. The relationships between $E_{\mathrm{r}}^{\prime}, E_{\mathrm{r}}^{\prime \prime}$ and $E^{\prime}, E^{\prime \prime}$ of the samples are

$$
\begin{gathered}
\frac{1}{E_{\mathrm{r}}^{\prime}}=\frac{\left(1-v_{\mathrm{i}}^{2}\right)}{E_{\mathrm{i}}}+\frac{\left(1-v_{\mathrm{s}}^{2}\right)}{E_{\mathrm{s}}^{\prime}}, \\
\frac{1}{E_{\mathrm{r}}^{\prime \prime}}=\frac{\left(1-v_{\mathrm{i}}^{2}\right)}{E_{\mathrm{i}}}+\frac{\left(1-v_{\mathrm{s}}^{2}\right)}{E_{\mathrm{s}}^{\prime \prime}}
\end{gathered}
$$

where $v$ is Poisson's ratio and the subscripts i and $\mathrm{s}$ refer to the indenter and the sample, respectively. The elastic modulus $E_{\mathrm{i}}$ of the diamond tip is $1140 \mathrm{GPa}$, which is much bigger than $E_{\mathrm{s}}^{\prime}(<30 \mathrm{GPa})$ and $E_{\mathrm{s}}^{\prime \prime}(<1 \mathrm{GPa})$ of the epoxy, and Poisson's ratio $v_{\mathrm{i}}$ of the diamond tip is 0.07 . By eq $2, E_{\mathrm{r}}^{\prime}$ and $E_{\mathrm{r}}^{\prime \prime}$ are approximately equal to $E_{\mathrm{s}}^{\prime}$ and $E_{\mathrm{s}}^{\prime \prime}$, respectively.

\section{RESULTS AND DISCUSSION}

\section{The Loss Tangent-Frequency Relation}

Figure 4 shows the variation of the loss tangent with the force frequency $(\omega / 2 \pi)$ at a mean contact force of $7 \mu \mathrm{N}$ (corresponding penetration depth is $8 \mathrm{~nm}$ ) using the Berkovich tip. The curve can be divided into three monotonically variation sections, and it distinctly reflects the nanoscale viscoelastic properties of the epoxy surface. The progressive increase of $\tan \delta$ in section $I$ is attributed to the increase of the

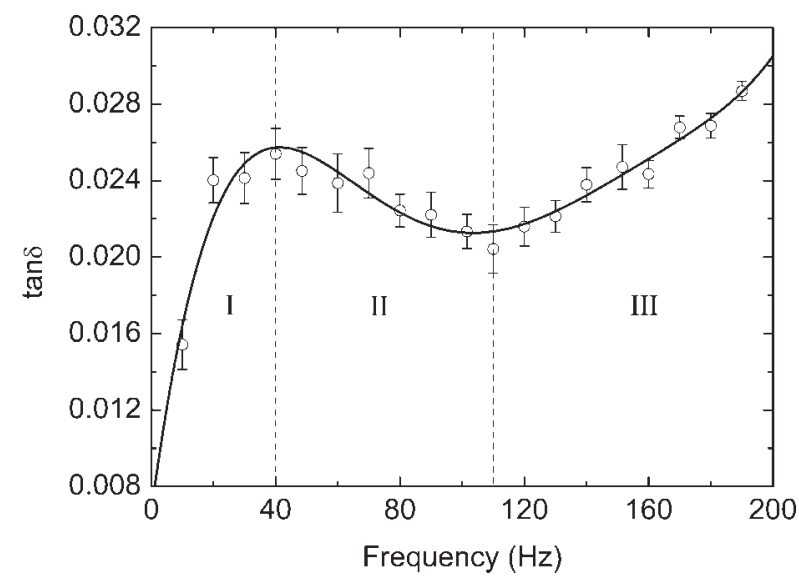

Figure 4. Loss tangent versus force frequency at the mean contact force of $7 \mu \mathrm{N}$.

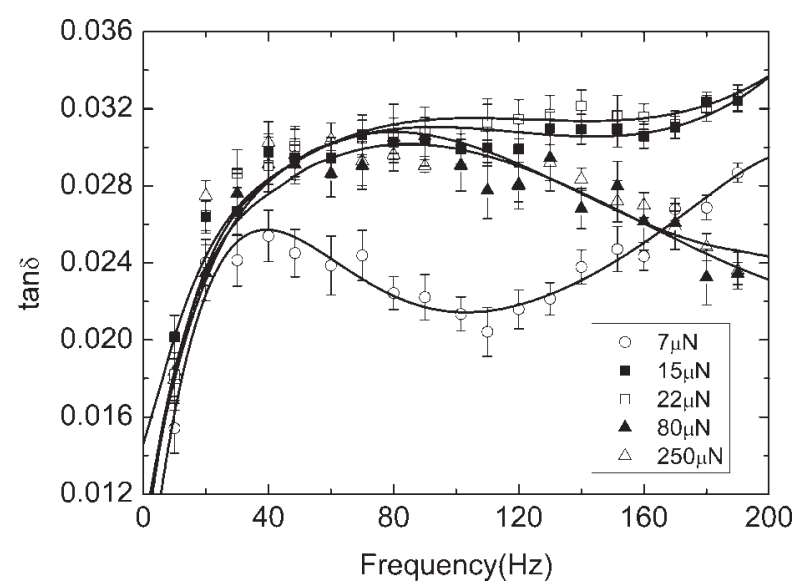

Figure 5. Loss tangent versus force frequency at different mean contact forces.

internal friction during the dynamic loading. This is due to the fact that the more the energy dissipates into the contact area, the larger the movement of the molecular chains and so the greater the internal friction among the molecular chains. The decrease of $\tan \delta$ in section II is associated with the decrease of the internal friction, which is because of the stiffening of the molecular configuration formed in section I. The first two sections are in good agreement with the classical viscoelastic phenomenon of polymers in a glassy state and at a constant temperature. ${ }^{3}$ At low frequency, $\tan \delta$ of crosslinked polymers maintains a small value. With an increasing frequency, $\tan \delta$ of all the amorphous polymers, whether crosslinked or not, reaches the maximum value rapidly and then goes back to a small value. Similar phenomena in some polyethylene surfaces have also been found through nano-DMA tests. ${ }^{6}$ Under high frequency (greater than $110 \mathrm{~Hz}$ in our case), the local molecular chains are agitated fiercely and some crosslinks between chains are possibly ruptured because of their large relative movements. So $\tan \delta$ increases again in section III. As the contact force rises, the dependence of $\tan \delta$ on the frequency is changed as shown in Figure 5. The variations of $\tan \delta$ at the force of 15 and $22 \mu \mathrm{N}$ (the corresponding penetration depth is 17 and $27 \mathrm{~nm}$ ) are similar to each other and the same situation is noticed for 80 and $250 \mu \mathrm{N}$ (the corresponding penetration depth is 75 and $170 \mathrm{~nm}$ ). Compared with $7 \mu \mathrm{N}$, the start frequency of section II and III is postponed for the contact force of 15 and $22 \mu \mathrm{N}$. It is noted that a constant force plateau appeared

Journal of Polymer Science: Part B: Polymer Physics DOI $10.1002 /$ polb 


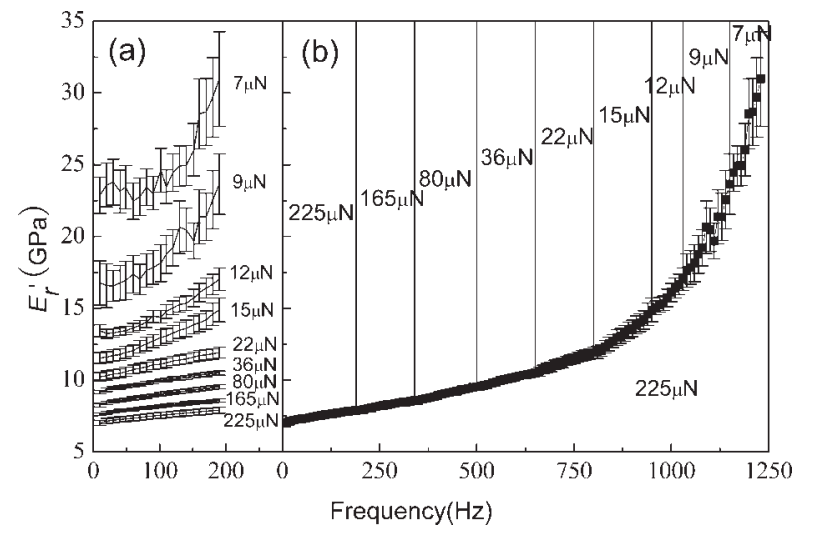

Figure 6. Dependence of the storage modulus on the force frequency. (a) Storage modulus, (b) master curve.

from about 80 to $150 \mathrm{~Hz}$, which marks the equilibrium between the dissipated energy and the internal friction of molecular chains. Above 150 $\mathrm{Hz}$, the rupture of crosslinks possibly happens and causes the increase of $\tan \delta$. When the contact force increases to 80 and $250 \mu \mathrm{N}$, the crosslinks rupture is not found during the rapid increase of the contact area, and so a continuous decrease of $\tan \delta$ is observed in the range of the adopted frequency.

\section{The Storage Modulus-Frequency Relation}

The gradual increase of the storage modulus with the frequency under different mean contact forces is presented in Figure 6(a). It is consistent with the polymer nature of dynamic storage in the glassy zone. ${ }^{3,8,9}$ As the force amplitude is kept constant, a higher frequency in the dynamic indentation means a higher loading rate. Even in quasi-static indentation, a higher loading rate has been found to cause a higher elastic modulus. ${ }^{30}$ The moduli measured by nano-DMA are larger than those measured by three-point bending (3.05 $\mathrm{GPa})$ and tension tests $(3.27 \mathrm{GPa})$ because of the effect of the loading rate.

The curves in Figure 6(a) are moved and superposed to construct a "master curve" as shown in Figure 6(b). With the "master curve" it is possible to evaluate the storage modulus under much higher frequency. As it is known, a larger indenting force brings a larger penetration depth, while the damping increases with the depth. With the "master curve," there exists an equivalence relation between force and frequency. So, the storage modulus of epoxy at one force level can be related to that at another one by a change in the frequency amplitude only. If this force-frequency dependence (similar to the time-temperature dependence) is valid, the method to construct the "master curve" of the storage modulus-frequency will be correct. From Figure 6(b), the storage modulus increases linearly with the frequency when the frequency is below $750 \mathrm{~Hz}$. Above this value, the increase of the storage modulus is accelerated more and more. This is possible because the length scale of polymer relaxation is proportional to the magnitude of the penetration depth or force. The curve of the storage modulus obtained with small penetration force can now be shifted to higher frequency.

\section{Effect of Tip Shape}

Figure 7 shows the effect of the indenting tip shape on the viscoelastic behavior of epoxy under two mean contact forces of $7 \mu \mathrm{N}$ and 250 $\mu \mathrm{N}$. The term of the tip shape includes both the tip geometry and the curvature radius of the tip top. For the Berkovich tip, there is a transition from sphere to sphere-tipped cone at a depth of about $10 \mathrm{~nm}$, considering the reported nominal radius and the apex angle. When the penetration depth is smaller than the transition depth, the Berkovich tip performs a spherical contact imposed on the sample. With further increasing of the penetration depth, the tip geometry will be of great influence. ${ }^{31}$ In Figure 7(a), the value of the loss tangent for two types of tips follows a quasi-similar variation in relation to frequency. However, the tip effect can still be recognized through careful analysis. The conospherical tip (the bigger tip) postpones the happening of both section II and section III. It means that the rupture of crosslinks is delayed due to the increase of the contact area with the big tip. By taking the data dispersion into account, the peak values of the loss tangent measured with a Berkovich indenter and a conospherical tip are not significantly different. It is observed in Figure 7(b) that the sharper tip (Berkovich tip) results in a larger storage modulus. Facility of plastic deformation is the chief reason for the effect of the tip radius on the storage modulus, according to Oyen and Philos. ${ }^{21}$ This means that the sharper tip tends to promote the initiation and evolution of 

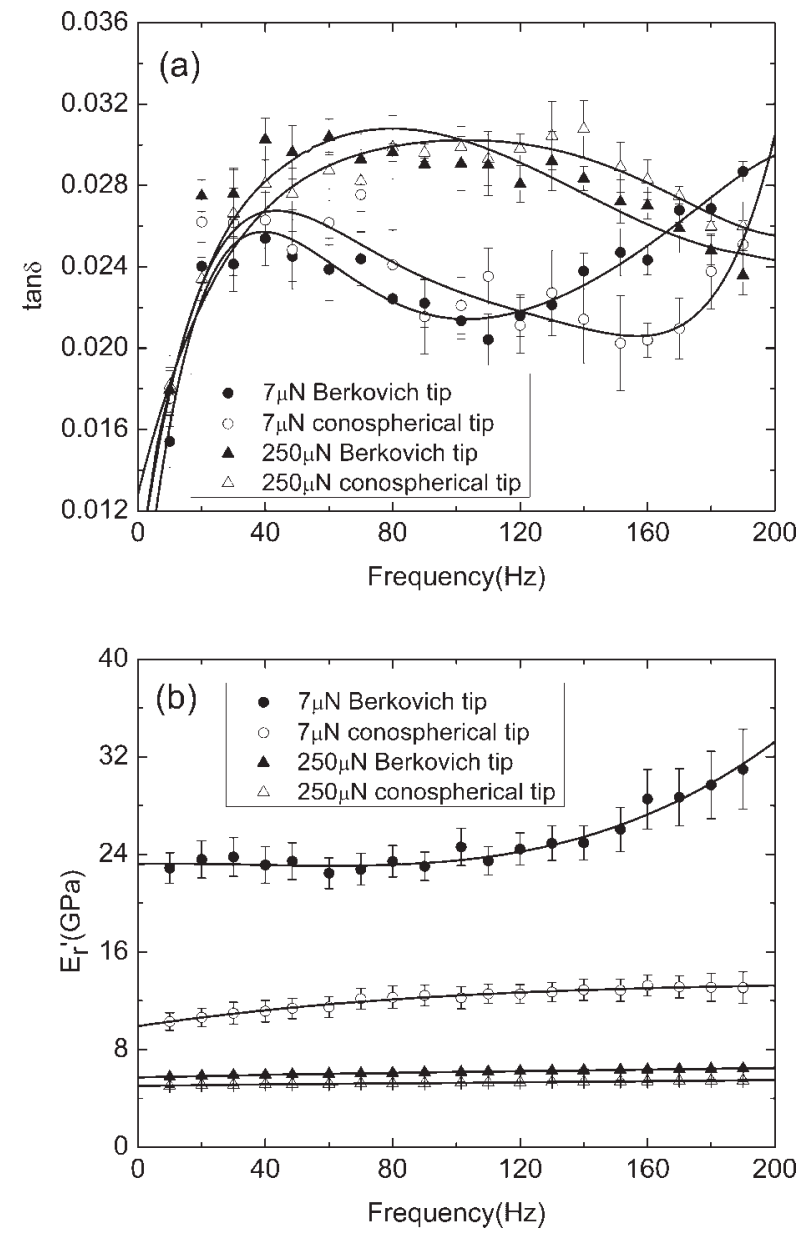

Figure 7. Viscoelastic properties versus force frequency with different tips. (a) Loss tangent, (b) storage modulus.

plastic deformation. At $7 \mu \mathrm{N}$, the difference of the storage modulus between the Berkovich tip and the conospherical tip is large. This is because of two reasons: the tip curvature radius and the surface effect both influence the measurement results. For a spherical contact under the same load, the small curvature radius will cause high stress. While at $250 \mu \mathrm{N}$, the geometry effect of the Berkovich tip becomes important, the storage modulus and loss tangent are only slightly different from the conospherical indenter. The stress possibly increases due to the imposition of the faceted pyramid edges on the epoxy surface.

\section{Continuous Stiffness Measurement}

CSM tests were specially performed to investigate the depth-dependent viscoelastic properties given in Figures 5 and 6 . Figure 8 shows that $\tan \delta$ fluctuates fiercely and $E_{\mathrm{r}}^{\prime}$ is very large when the depth is below $30 \mathrm{~nm}$. With the penetration depth increasing, $\tan \delta$ varies in a narrow band and $E_{\mathrm{r}}^{\prime}$ progressively decreases to a quasi-constant value. This phenomenon was observed in various types of polymers ${ }^{6,14,18,32}$ and explained to be attributed to the crosslink density-depth relation. ${ }^{33}$ Moreover, some experimental factors were also mentioned as follows: localized oxidation, wetting and aging of the very near-to-surface features (caused by exposing the polymers to light and air for some time before the experiments or during fabrication), inaccurate contact area (caused by roughness of the sample surfaces and imprecise tip area function), size effect, and measurement errors on nanoscale experiments.

Figure 8 shows that the smaller the penetration depth, the greater is the effect of tip shape.
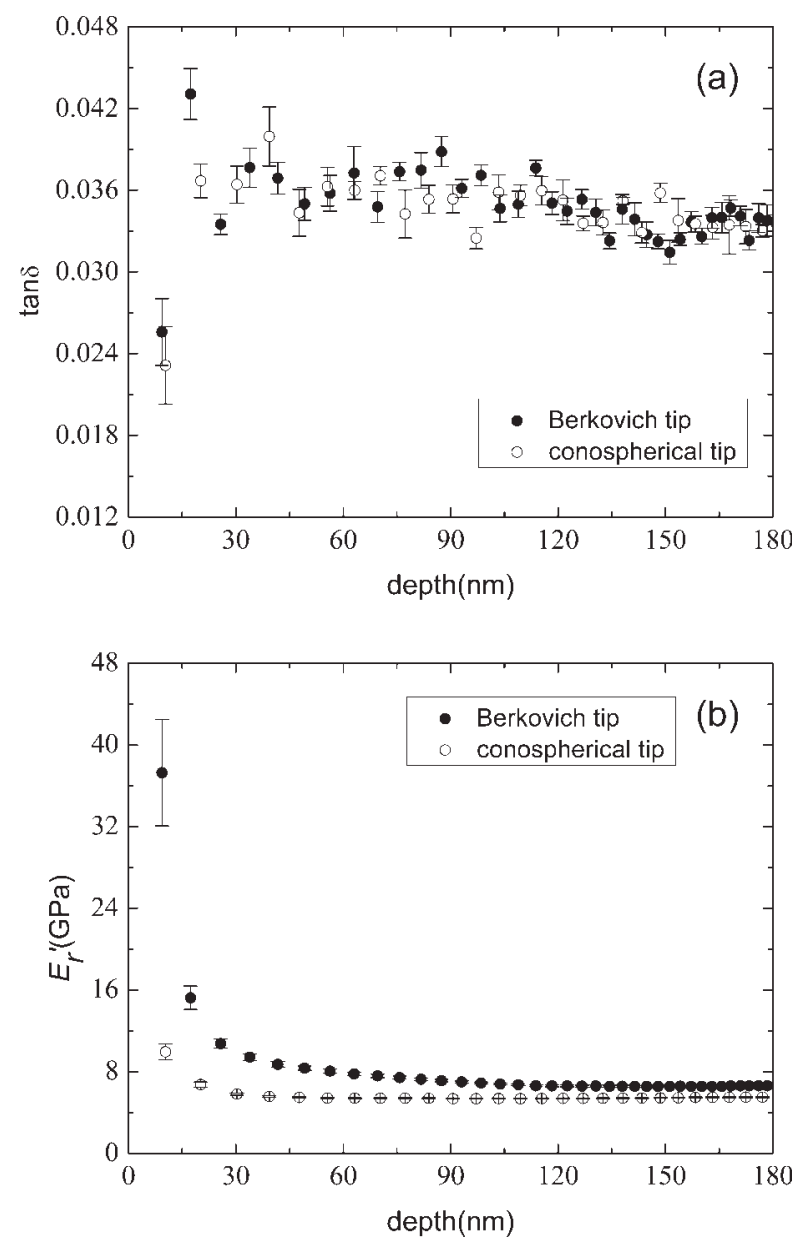

Figure 8. Viscoelastic properties versus penetration depth at $75 \mathrm{~Hz}$. (a) Loss tangent, (b) storage modulus.

Journal of Polymer Science: Part B: Polymer Physics DOI $10.1002 /$ polb 
At a large penetration depth (larger than 120 $\mathrm{nm}$ ), the results from the use of the Berkovich and the conospherical tips show an approximately constant difference. This can also be analyzed with the theoretical function of a projected contact area $A_{c}$, which is related to the surface roughness, pile-up, or sink-in on an indent boundary and an imprecise tip radius. The contact area function mostly used is based on the testing of hard materials. Though some progress has been made on the correction factor for the contact area, ${ }^{34}$ there is still a lot of work to do. By eq $1, \tan \delta$ is independent of the tip shape and the contact area function. Therefore, the values should be very close for both two tips.

\section{CONCLUSIONS}

This work demonstrates the effect of force frequency, penetration depth, and tip shape on the viscoelastic properties of a cycloaliphatic epoxy by nano-DMA experiments.

With the increase of force frequency, the loss tangent manifests a characteristic of a three-section variation, while the storage modulus increases monotonically. Both the loss tangent and the storage modulus are influenced by the level of contact force. A "master curve" of the storage modulus as a function of the force frequency is established to describe the dynamic behavior of the epoxy surface under super frequency.

The CSM tests reveal the depth-dependent viscoelastic behavior of the epoxy surface: both the storage modulus and the loss tangent are quite scattered when the penetration depth is below $30 \mathrm{~nm}$. Over $120 \mathrm{~nm}$, both of them become quasi-stable and representative.

The viscoelastic properties measured by means of the Berkovich and the conospherical tips present a similar relation to frequency as well as to depth. A small difference of the storage modulus exists between the Berkovich and the conospherical tips. However, the loss tangent seems to be independent of the tip shape. Our study also shows that to get a stable and valuable storage modulus and loss tangent irrespective of the different tips, the penetration depth should be larger than $120 \mathrm{~nm}$.

This work was supported by the Natural Science Foundation of China (NSFC) (grant number: 10472002).
The authors appreciate nanoresins AG (hanse chemie AG) which kindly provided the specimens.

\section{REFERENCES AND NOTES}

1. Ellis, B. Chemistry and Technology of Epoxy Resins; Blackie Academic and Professional: Glasgow, 1993; Chapter 2, pp 37-71.

2. Chan, C. M. Polymer Surface Modification and Characterization; Hanser Publications: New York, 1994; Chapter 1, pp 1-34.

3. Ferry, J. D. Viscoelastic Properties of Polymers; Wiley: New York, 1980; Chapter 15, pp 444448.

4. Kajiyama, T.; Tanaka, K.; Ohki, I.; Ge, S. R.; Yoon, J. S.; Takahara, A. Macromolecules 1994, 27, 7932-7934.

5. Krupicka, A.; Johansson, M.; Hult, A. Macromol Mater Eng 2003, 288, 108-116.

6. Chakravartula, A.; Komvopoulos, K. Appl Phys Lett 2006, 88, 131901.

7. Loubet, J. L.; Oliver, W. C.; Lucas, B. N. J Mater Res 2000, 15, 1195-1198.

8. White, C. C.; Vanlandingham, M. R.; Drzal, P. L.; Chang, N. K.; Chang, S. H. J Polym Sci Part B: Polym Phys 2005, 43, 1812-1824.

9. Tsukruk, V. V.; Gorbunov, V. V.; Huang, Z.; Chizhik, S. A. Polym Int 2000, 49, 441-444.

10. Wahl, K. J.; Asif, S. A. S.; Greenwood, J. A.; Johnson, K. L. J Colloid Interface Sci 2006, 296, 178188.

11. Groot, R. D.; Agterof, W. G. M. Macromolecules 1995, 28, 6284-6295.

12. Gurtovenko, A. A.; Gotlib, Y. Y. Macromolecules 2000, 33, 6578-6587.

13. Bouaita, N.; Bull, S. J.; Palacio, J. F.; White, J. R. Polym Eng Sci 2006, 46, 1160-1172.

14. VanLandingham, M. R. J Res Natl Inst Stand Technol 2003, 108, 249-265.

15. Oliver, W. C.; Pharr, G. M. J Mater Res 1992, 7, 1564-1583.

16. Oliver, W. C.; Pharr, G. M. J Mater Res 2004, 19, $3-20$.

17. Sneddon, I. N. Int J Eng Sci 1965, 3, 47-57.

18. Briscoe, B. J.; Fiori, L.; Pelillo, E. J Phys D Appl Phys 1998, 31, 2395-2405.

19. Feng, G.; Ngan, A. H. W. J Mater Res 2002, 17, $660-668$.

20. Oyen, M. L.; Cook, R. F. J Mater Res 2003, 18, 139-150.

21. Oyen, M. L. Philos Mag 2006, 86, 5625-5641.

22. Shimizu, S.; Yanagimoto, T.; Sakai, M. J Mater Res 1999, 14, 4075-4086.

23. Cheng, L.; Xia, X.; Yu, W.; Scriven, L. E.; Gerberich, W. W. J Polym Sci Part B: Polym Phys 2000, 38, 10-22.

24. Sakai, M. Philos Mag A 2002, 82, 1841-1849. 
25. Cheng, Y. T.; Ni, W. Y.; Cheng, C. M. Phys Rev Lett 2006, 97, 075506.

26. Fischer-Cripps, A. C. Introduction to Contact Mechanics; Springer: New York, 2000; Appendix 1, pp 174-178.

27. Jeng, Y. R.; Tan, C. M. Appl Phys Lett 2006, 89, 251901.

28. Li, X.; Bhushan, B. Mater Charact 2002, 48, 1136.

29. Zhang, H.; Zhang, Z.; Friedrich, K.; Eger, C. Acta Mater 2006, 54, 1833-1842.
30. Fang, T. H.; Chang, W. J. Microelectron J 2004, 35, 595-599.

31. Briscoe, B. J.; Sebastian, K. S.; Adams, M. J. J Phys D Appl Phys 1994, 27, 1156-1162.

32. Asif, S. A. S.; Wahl, K. J.; Colton, R. J Rev Sci Instrum 1999, 70, 2408-2413.

33. Gu, X.; Raghavan, D.; Ho, D. L.; Sung, L.; VanLandingham, M. R.; Nguyen, T. Mater Res Soc Symp Proc 2002, 710, DD 10.9.1-6.

34. Troyon, M.; Huang, L. Y. J Mater Res 2005, 20, $610-617$. 